\title{
Effective asexual reproduction of a widespread soft coral: Comparative assessment of four different fragmentation methods
}

\author{
Sohyoung Kim ${ }^{\text {Corresp., } 1}$, Christian Wild ${ }^{1}$, Arjen Tilstra ${ }^{1}$ \\ 1 Marine Ecology Department, Faculty of Biology and Chemistry, Universität Bremen, Bremen, Germany \\ Corresponding Author: Sohyoung Kim \\ Email address: soh_kim@uni-bremen.de
}

Background. Many coral reefs worldwide are experiencing declines in hard corals, resulting in other benthic organisms, such as soft corals, becoming more dominant. As such, more studies on the ecophysiology of soft corals are needed. Despite many methods for asexual reproduction of hard corals, effective methods for soft corals, i.e., without a hard skeleton, are scarce. This study, thus, assessed four fragmentation methods, the glue, rubber band, tunnel mesh, and plug mesh method for the pulsating soft coral Xenia umbellata that is widely distributed in the tropical Indo-Pacific.

Methods. Methods were comparatively assessed by determining the required time and labor for the fragmentation plus the health status of the fragmented corals by measuring their oxygen fluxes and pulsation rates, i.e., a special feature of this soft coral that can be used as a proxy for its health.

Results. There were no significant health status differences between methods. This was indicated by similar gross photosynthesis (between 7.4 to $9.7 \mu \mathrm{g} \mathrm{O}_{2}$ polyp $\mathrm{p}^{-1} \mathrm{~h}^{-1}$ ) and pulsating rates (between 35 and 44 pulses $\mathrm{min}^{-1}$ ) among methods. In terms of time/labor intensity and success rates, i.e., the percentage of fragments attached to the desired surface, the plug mesh method was the most efficient method with a significantly higher success rate (95 $\pm 5 \%$ ), while the others had a success rate between $5 \pm 5$ and 45 $\pm 15 \%$. The time needed for fragmentation, though not significant, was also the shortest (78 $\pm 11 \mathrm{sec}$ fragment ${ }^{-1}$ ), while other methods required between $84 \pm 14$ and $126 \pm 8 \mathrm{sec} \mathrm{frag}^{-1}$. The plug mesh method may thus be a valuable tool related to the reproduction of soft corals for use in subsequent experimental work. 


\section{Effective asexual reproduction of a widespread soft coral:}

\section{Comparative assessment of four different fragmentation}

\section{3 methods}

5 Sohyoung Kim ${ }^{1}$, Christian Wild ${ }^{1}$, Arjen Tilstra ${ }^{1}$

6 'Marine Ecology Department, Faculty of Biology and Chemistry, University of Bremen,

7 Leobener Str. 6 (UFT), 28865 Bremen, Germany

9 Corresponding author:

10 Sohyoung $\mathrm{Kim}^{1}$

11 Leobener Str. 6 (UFT), 28865 Bremen, Germany

12 Email address: soh_kim@uni-bremen.de

14 Abstract

15 Background. Many coral reefs worldwide are experiencing declines in hard corals, resulting in

16 other benthic organisms, such as soft corals, becoming more dominant. As such, more studies on

17 the ecophysiology of soft corals are needed. Despite many methods for asexual reproduction of

18 hard corals, effective methods for soft corals, i.e., without a hard skeleton, are scarce. This study,

19 thus, assessed four fragmentation methods, the glue, rubber band, tunnel mesh, and plug mesh

20 method for the pulsating soft coral Xenia umbellata that is widely distributed in the tropical

21 Indo-Pacific. 
22 Methods. Methods were comparatively assessed by determining the required time and labor for

23 the fragmentation plus the health status of the fragmented corals by measuring their oxygen

24 fluxes and pulsation rates, a special feature of this soft coral that can be used as a proxy for its

25 health.

26 Results. There were no significant health status differences between methods. This was indicated

27 by similar gross photosynthesis (between 7.4 to $9.7 \mu \mathrm{g} \mathrm{O}_{2}$ polyp $^{-1} \mathrm{~h}^{-1}$ ) and pulsating rates

28 (between 35 and 44 pulses min $^{-1}$ ) among methods. In terms of time/labor intensity and success

29 rates, i.e., the percentage of fragments attached to the desired surface, the plug mesh method was

30 the most efficient method with a significantly higher success rate $(95 \pm 5 \%)$, while the others

31 had a success rate between $5 \pm 5$ and $45 \pm 15 \%$. The time needed for fragmentation, though not

32 significant, was also the shortest $\left(78 \pm 11 \mathrm{sec}\right.$ fragment $\left.^{-1}\right)$, while other methods required between

$3384 \pm 14$ and $126 \pm 8 \mathrm{sec} \mathrm{frag}^{-1}$. The plug mesh method may thus be a valuable tool related to the

34 reproduction of soft corals for use in subsequent experimental work.

35

36 Keywords: soft coral research, asexual reproduction, fragmentation methods

\section{Introduction}

39 Coral reefs are high biodiversity ecosystems that provide services such as coastal protection,

40 food provision, and tourism which maintain the livelihoods of millions of people (Moberg \&

41 Folke, 1999; Reaka-Kudla and Wilson, 1997; Woodhead et al., 2019). However, these complex

42 ecosystems have been declining for decades due to global and local anthropogenic stressors

43 (De'Ath et al., 2012; Naumann et al., 2015; Hoegh-Guldberg, Pendleton \& Kaup, 2019). These

44 stressors can trigger a so called 'phase shift', where benthic dominance shifts from hard corals 
45 towards algae-, sponge-, or soft coral dominance (Done, 1992; McClanahan, Cokos \& Sala,

46 2002; Maliao, Turingan \& Lin, 2008; Wood \& Dipper, 2008; Wild et al., 2011; Baum et al.,

47 2016). Although phase shifts from hard coral to (macro)algae are studied relatively often,

48 research on the shift towards soft coral dominance are more scarce (Norström et al., 2009; Cruz

49 et al., 2016).

50 Though soft coral communities are rising in numbers and softs corals becoming the

51 dominant organism on many reefs, there is a lack of studies exploring the ecological impact of

52 these communities. One study by Wood and Dipper (2008) indicated that some soft corals, like

53 xeniids, could decrease the overall biodiversity by not only blocking the settlement and

54 recruitment of hard corals, but also by overgrowing and eventually killing the remaining ones.

55 Not only are they considered invasive due to their high fecundity and long reproductive season

56 (Wood \& Dipper, 2008), xeniid soft corals are also more resilient to global and local stressors

57 (Wild et al., 2011; Vollstedt et al., 2020). This could intensify the phase shift from hard coral

58 towards soft coral dominance. As the effects of these shifts are yet to be established, there is an

59 urgent need for more relevant studies assessing soft coral ecophysiology to better understand and

60 manage this benthic functional group accordingly.

61 When conducting research with soft corals, however, securing sufficient numbers of coral

62 samples is one of the first yet highly time-consuming procedures which could span weeks to

63 months depending on the number of samples. As the samples often need to be similar in size and

64 should be easy to relocate, i.e., should be attached to a small rock or an independently moveable

65 substrate such as a coral plug, many ex-situ experiments utilize a method called 'fragmentation'.

66 While there are many detailed and straightforward fragmentation protocols for hard corals, i.e.,

67 corals that build a hard calcium carbonate $\left(\mathrm{CaCO}_{3}\right)$ skeleton (Johnson et al., 2011; Forsman et 
68 al., 2015), it is difficult to adopt the same methods for corals that lack a hard skeleton, i.e., soft

69 corals (Veron, 2011). Though some soft corals have a support system that would allow the use of

70 the same methods as for hard corals, e.g., tough leathery tissue as associated with coral genera

71 such as Sinularia and Sarcophyton, or gorgonians which are supported by a gorgonin skeleton,

72 many other soft corals lack any rigid support, such as the widespread xeniid soft corals. These

73 soft corals, despite being a successful colonizer (Benayahu \& Loya, 1985), may need special

74 methods as current approaches can be time- and labor-intensive when preparing hundreds of

75 similar sized fragments with minimal disturbances. While much information exists in grey

76 literature and aquarist exchange platforms, there are a limited number of scientific papers

77 exploring the topic of fragmentation for these types of soft corals (Ellis \& Sharron, 1999). Some

78 studies by Barton et al. (2017), Borneman and Lowrie (2001), and Chaitanawisuti \&

79 Kritsanapuntu (2019) do touch upon soft coral fragmentation methods, but lack a method for the

80 corals without any rigid support system.

81 Thus, the aim of this study is to (i) assess and compare four fragmentation methods for

82 the pulsating soft coral, the Xenia umbellata, i.e., the glue, rubber band, tunnel mesh, and plug

83 mesh method, and (ii) provide a protocol for the best method. Among the four methods, the glue

84 and rubber band methods have already been widely used in laboratories (e.g., Barton et al. 2017;

85 Ellis \& Sharron, 1999). In contrast, the tunnel mesh and plug mesh methods were modified from

86 a recently patented method named the tunnel cube method by Curry (2020). We assessed the

87 methods by ranking them in four evaluations: success rate, time efficiency, oxygen fluxes, and

88 pulsating rate. The first two were used to determine the labor/time efficiency and the latter two

89 were used to assess the health. After assessments, optimization of the best method focused on 
90 finding the minimal time needed for fragmentation recovery and thus, to provide a useful

91 protocol for optimized soft coral fragmentation.

92

\section{2. Materials and methods}

\section{$94 \quad 2.1$ Sample species and reproduction methods}

95 The soft coral used in the experiment, Xenia umbellata (Lamarck, 1816), originated from the 96 northern Red Sea and has been kept in recirculating holding tanks for approximately 3 years.

97 Four methods were tested in this experiment, i.e., the glue method, rubber band method,

98 tunnel mesh method, and plug mesh method as described below. Large colonies were carefully

99 collected from the holding tank and fragmented into smaller sizes in a temporary water basin

100 filled with water from the holding tank. To minimize the stress on fragments, no more than five

101 fragments were treated at the same time in the water basin. Once five fragments were ready, they

102 were attached on calcium carbonate coral plugs (Aquaforest $₫$ AF Plug Rocks cuttings) or placed

103 in egg crates depending on the method and placed into designated tanks immediately. After the

104 water in the basin was replaced, this procedure was repeated until the needed number of

105 fragments was fulfilled. All fragmentation procedures and further assessments (see 2.3.) were

106 conducted by a single individual.

107

108 2.1.1. The glue method

109 One coral fragment was glued to the center of a dry coral plug using a few drops of coral glue

110 (Microbe-Lift Coralscaper seconds glue). The lower part of the cut coral was embedded into the

111 glue substrate (Fig. S1) using tweezers and left to harden for a few seconds in the water basin

112 before being transferred to its respective tank. 


\section{2.1.2. The rubber band method}

115 Each coral fragment was attached to the coral plug using two rubber bands, with a diameter of 2

$116 \mathrm{~cm}$ when unstretched, crossing each other making an ' $\mathrm{X}$ ' shape on the plug (Fig. S2). Rubber

117 bands were applied as loose and gently as possible to minimize the stress caused on fragments by

118 letting minimum force to keep the fragments onto the plugs. Fragments were then transferred to

119 respective tanks immediately after attachment.

120

\section{2.1.3. The tunnel mesh method}

122 Adapted from the tunnel cube method by Curry (2020), the tunnel mesh method was

123 composed of two mesh fabrics and two egg crate grids held together with 2-4 rubber bands (Fig.

124 1A). The mesh fabrics had a diameter of $3.5 \mathrm{~mm}$ (Large mesh: Mesh L) and $2.0 \mathrm{~mm}$ (Small

125 mesh: Mesh S) per opening. Different mesh fabrics can be used as long as they serve their

126 purpose. The top mesh (Mesh L) needs to keep the coral fragments inside the egg crate tunnel

127 while maximizing the water flow. The bottom mesh screen (Mesh S) needs to have a mesh size

128 small enough for the fragments to attach while subsequently allowing water to flow through. A

129 fragmented coral was placed into one egg crate tunnel and closed off with Mesh L (Fig. 1A).

130 After assembling all the components with rubber bands, the tunnel mesh grid was put in its

131 respective tank. Coral fragments were placed in egg crate tunnels without fragments in

132 neighboring tunnels to prevent physiological interference to each other.

133 In the beginning of the experiment, to keep the tunnel mesh method assembly from

134 floating, empty plugs were placed on top which later on served as control plugs to measure the

135 blank control in oxygen flux assessments (see 2.3.3.). 


\section{2.1.4. The plug mesh method}

138 Also adapted from the tunnel cube method by Curry (2020), the plug mesh method required two 139 egg crate grids, one mesh fabric (Mesh L) and five coral plugs (Fig.1B). Five coral plugs were

140 evenly placed in the lower egg crate to hold the plugs with another egg crate placed on top of the

141 plugs to hold the coral fragments. One fragmented coral was placed inside the top egg crate

142 tunnel covering the coral plug. Once all fragments were in place, a Mesh L fabric was placed to

143 cover the structure, which was subsequently fixed with rubber bands. The whole assembly was

144 then transferred to its respective tank. Fragment arrangement was identical to the tunnel mesh 145 method described previously.

148 Fig. 1. Illustration of (A) tunnel mesh method and (B) plug mesh method. All components are

149 fixed with two to four rubber bands on the sides. An extra egg crate is an important factor so that

150 there can be water circulating through the egg crate holes. Once the coral fragment is attached to

151 the mesh fabric or plug beneath, the egg crate and mesh fabric on top can be removed. Mesh

152 fabrics are differentiated by diameter size of $3.5 \mathrm{~mm}$ (Large mesh: Mesh L) and $2.0 \mathrm{~mm}$ (Small 153 mesh: Mesh S) per opening.

\subsection{Experimental design and maintenance}

157 We used four tanks with a total water volume of approximately 501 each. After filling the tanks

158 with water from the holding tank, they were left to stabilize for $48 \mathrm{~h}$. A total of 80 fragments 
159 were evenly divided over the four tanks. Thus, each tank contained a total of 20 newly

160 fragmented corals (five fragments $\mathrm{x}$ four methods) with two additional control corals originating

161 from the same holding tank as the newly fragmented corals.

162 Water parameters were then maintained at the following levels, similar to the holding

163 tank, throughout the experiment: salinity $34-36 \mathrm{PSU}$, temperature $25-26.5^{\circ} \mathrm{C}, \mathrm{pH} 8.26 \pm 0.02$,

164 calcium $415 \pm 5 \mathrm{mg} \mathrm{L}^{-1}$, magnesium $1230 \pm 19.64 \mathrm{mg} \mathrm{L}^{-1}$, nitrite $<0.01 \mathrm{mg} \mathrm{L}^{-1}$, nitrate $<0.5 \mathrm{mg}$

$165 \mathrm{~L}^{-1}$, ammonium $<0.05 \mathrm{mg} \mathrm{L}^{-1}$, phosphate $<0.02 \mathrm{mg} \mathrm{L}^{-1}$. Water flow $(300 \mathrm{~L} / \mathrm{h})$ was provided by

166 EHEIM CompactOn 300 pumps (EHEIM GmbH and Co. KG, Germany). Light was provided by

167 daytime LED light (WALTRON daytime ${ }^{\circledR}$ LED light, Germany) in a 9:15 h light:dark cycle with

168 an intensity of $\sim 55 \pm 5 \mu \mathrm{mol} \mathrm{m} \mathrm{m}^{-2} \mathrm{~s}^{-1}$. Approximately $10 \%$ of water was exchanged each day with

169 water from the holding tank. Coral fragments were fed with coral food (REEF-ROIDS ${ }^{\mathrm{TM}}$

170 Engineered Coral Food, Polyp Lab, Canada) two to three times per week.

$171 \quad$ For the optimization process, we used three tanks with a total of 15 newly fragmented

172 corals. Each tank had five fragments and two controls from the same holding tank. All the other

173 parameters were identical as the main experiment.

174

\section{2.3. Reproduction method assessment categories}

176 We based our assessment of these four methods on (1) success rate, (2) fragmentation time, (3)

177 oxygen fluxes, and (4) pulsating rate. Success rate, defined as the attachment percentage to the

178 desired surface of each method, and fragmentation time were used to determine the time- and

179 labor- efficiency of each method, while oxygen fluxes and pulsating rate measurements were

180 used as a proxy for the health status (McGillis et al., 2009; Jantzen et al., 2013; Vollstedt et al.,

181 2020). The method with the best results was then further optimized using three assessment 
182 categories: (1) success rate, (2) oxygen fluxes, and (3) pulsating rate. Compared to the main

183 experiment, assessments were conducted in a more detailed timeline to provide a better

184 understanding and thus, to optimize the best method.

185

186 2.3.1. Success rate

187 Success rate was assessed and calculated on the eighth day of post fragmentation as there were

188 no further changes observed nor made to the fragments after the eighth day, e.g., cutting rubber

189 bands or scraping of egg crates.

190 For the tunnel mesh and plug mesh method, a successful attachment was counted when it

191 was attached to the mesh or plug, respectively, not the egg crate. Also, it was considered to be a

192 successful attachment only if the coral fragment remained attached to the mesh or plug under

193 ambient water flow without support from the rubber band or egg crate tunnel. If fragments

194 attached to the egg crate walls, they were gently scraped off and placed back on the mesh fabric

195 or plug with the polyps facing upwards to stimulate attachment. Note: Failure rates do not

196 represent mortality. Rather, they indicate that the attachment to the desired surface has failed.

197

198 2.3.2. Fragmentation time

199 While fragmenting the corals, the time needed to produce one fragment assembly was recorded.

200 This included the time for cutting, mounting, and placing one fragment assembly into its

201 designated tank.

202

203 2.3.3. Oxygen fluxes 
204 Measurements were done on the twelfth-day post fragmentation using 19 jars (total=19, 3 per

205 method, 3 controls, 3 empty plugs as blank control, and 1 with only water to measure

206 background oxygen evolution/depletion).

207 Coral fragments were incubated for $1 \mathrm{~h}$ in light to assess net photosynthesis $\left(P_{\text {net }}\right)$ and $1 \mathrm{~h}$ in dark 208 conditions to assess respiration $\left(R_{\text {dark }}\right)$. Coral fragments were transferred into individual $160 \mathrm{ml}$ 209 glass jars filled with the same water from where the coral came from containing a small magnet 210 for stirring. Right after one fragment was transferred into a jar without any air contact, the time,

211 temperature, and oxygen concentration were measured using an optode sensor (Hach

212 IntelliCAL/Optical Dissolved Oxygen Probe or similar) and then sealed air-tight making sure

213 there were no air bubbles inside. Jars were placed in a water bath kept at $26^{\circ} \mathrm{C}$ which was placed 214 on top of a magnetic stirring machine (Poly 15, Thermo Scientific VARIOMAG® Magnetic

215 Stirrers) set at $190 \mathrm{rpm}$ to prevent a gradient of oxygen forming inside the jars. After the $1 \mathrm{~h}$

216 incubation, the lid was opened carefully and the temperature and oxygen level were measured 217 again. The difference in oxygen concentration between start and finish was used to calculate the 218 oxygen fluxes. Data was corrected for controls, and normalized to incubation time and number of 219 polyps per sample resulting in fluxes presented in " $\mu \mathrm{g} \mathrm{O}_{2}$ polyp $^{-1} \mathrm{~h}^{-1}$ ". Light incubations were 220 performed under the same light conditions as in the respective tanks while dark incubations were 221 performed in complete darkness. Gross photosynthesis $\left(P_{\text {gross }}\right)$ was calculated using the following 222 equation: $P_{\text {net }}=P_{\text {gross }}+R_{\text {dark }}$ (e.g., Jantzen et al., 2013; Tilstra et al., 2019). Oxygen flux 223 measurements for optimizing the best method were measured on the seventh-day post 224 fragmentation using 10 jars (total $=10,3$ controls, 3 respective method, 3 empty plugs as blank 225 control, and 1 with only water to measure background). 


\section{2.3.4. Pulsating rate}

228 Pulsating rates were assessed by counting pulsation of three randomly selected polyps per

229 fragment for $30 \mathrm{sec}$. This was then calculated to give a mean value of the number of pulsations

230 per minute (pulses min $^{-1}$ ). A full contraction and opening of all polyp tentacles were considered

231 as one pulse. Pulsating rates were assessed every $48 \mathrm{~h}$ at a fixed time to avoid differences due to 232 circadian rhythms.

233 In the optimization of the best method, measurements were done every $24 \mathrm{~h}$ for 7 -days to 234 provide more detailed information.

235

236

\subsection{Statistical analyses}

237 Statistical analyses were performed using Sigmaplot 14.5 and R Studio for Windows. All data 238 are presented as means \pm SE. Differences between treatments were analyzed for significance by 239 one-way analysis of variance (ANOVA) using "method" as the main factor. Percentage data 240 were transformed by applying the arcsine square root transformation for the one-way ANOVA 241 test. Post-hoc analysis (Tukey’s HSD) was carried out when significant differences were found.

242 Asterisks indicate significant differences between methods $\left({ }^{*} p<0.01,{ }^{*} p<0.001\right)$.

\section{3. Results}

\subsection{Main experiment}

\subsubsection{Success rate}

248 The glue method had the lowest success rate of $5 \pm 5 \%$, while the rubber band and tunnel mesh 249 method had a success rate of $45 \pm 5 \%$ and $45 \pm 15 \%$, respectively (Fig. 2A). The plug mesh 
250 method had a significantly higher success rate compared to the other three methods $(95 \pm 5 \%$;

251 one-way ANOVA, $\mathrm{F}_{[3,12]}=21.75, p<0.001 ;$ Fig. $\left.2 \mathrm{~A}\right)$.

252

253 3.1.2. Fragmentation time

254 The time needed to assemble one coral fragment was not statistically significant (one-way 255 ANOVA, $\left.\mathrm{F}_{[3,12]}=3.17, p=0.064\right)$. The glue method needed $96 \pm 14 \mathrm{sec} \mathrm{frag}^{-1}$ while it took the 256 longest for the rubber band method of $126 \pm 8 \mathrm{sec}_{\text {frag- }}{ }^{-1}$ (Fig. 2B). The tunnel mesh method took $25784 \pm 14 \mathrm{sec}$ frag $^{-1}$ while the plug method required the shortest time, $78 \pm 11 \mathrm{sec}$ frag $^{-1}$, to produce 258 one fragment assembly (Fig. 2B).

261 Fig. 2. (A) Attachment success rate and (B) fragmentation time of four different fragmentation 262 methods as an assessment of time- and labor-efficiency. The four methods are the glue (Glue), 263 rubber band (Rubber), tunnel mesh (Tunnel), and plug mesh method (Plug). Values are given as 264 means $\pm \mathrm{SE}$ of $\mathrm{N}=3-4$ replicates. Different letters $(\mathrm{a}, \mathrm{b})$ indicate significant differences between 265 the methods (one-way ANOVA, $\mathrm{F}_{[3,12]}=21.75, p<0.001$ ).

266

267 3.1.3. Oxygen fluxes

268 There were no statistically significant differences between the control and each method for all 269 three parameters measured, i.e., $P_{\text {gross }}$ (one-way ANOVA, $\mathrm{F}_{[3,8]}=0.45, p=0.725$ ), $P_{\text {net }}$ (one-way 270 ANOVA, $\mathrm{F}_{[3,8]}=0.36, p=0.786$ ), and $R_{\text {dark }}$ (one-way ANOVA, $\mathrm{F}_{[3,8]}=0.84, p=0.509$ ) (Fig.

$2713 \mathrm{~A}$ ). Mean values ranged between 7.43 to $9.73 \mu \mathrm{g} \mathrm{O} \mathrm{O}_{2}$ polyp $^{-1} \mathrm{~h}^{-1}$ for $P_{\text {gross }}, 3.25$ to $4.95 \mu \mathrm{g} \mathrm{O}$ 272 polyp $^{-1} \mathrm{~h}^{-1}$ for $P_{\text {net }}$ and -4.88 to $-3.68 \mu \mathrm{g} \mathrm{O}_{2}$ polyp $^{-1} \mathrm{~h}^{-1}$ for $R_{\text {dark }}$ (Fig. $3 \mathrm{~A}$ ). 
274 attached to the plug.

275

276

3.1.4. Pulsating rate

277 With the exception of day 8 (one-way ANOVA, $\mathrm{F}_{[4,12]}=9.76, p<0001$ ), overall pulsating rates 278 differed in every measurement day without significant differences among methods. Among the 279 methods, the plug mesh method had significantly higher pulsating rates than the control and 280 rubber band method (Tukey's HSD, $p<0.001, p<0.01$, respectively). All methods had lower 281 average pulsating rates in the beginning of the experiment which gradually increased. On the 282 second day, the mean pulsating rate of all methods was $27.13 \pm 1.01$ pulses min $^{-1}$ while from day 2834 to 12 , the mean pulsating rate of the three methods was in the range of 35.99 to 44.06 pulses $284 \min ^{-1}$ (Fig. 3B).

285 The glue method was not assessed here as only one out of 20 replicates successfully 286 attached to the plug.

289 Fig. 3. (A) Oxygen fluxes and (B) pulsating rates measured on the twelfth-day post

290 fragmentation as health assessments of three different fragmentation methods. The three methods 291 are the rubber band (rubber), tunnel mesh (tunnel), and plug mesh method (plug). $P_{\text {gross }}=$ gross 292 photosynthesis, $P_{\text {net }}=$ net photosynthesis, $R_{\text {dark }}=$ dark respiration. Values are given as means \pm $293 \mathrm{SE}$ of $\mathrm{N}=3-4$ replicates. Asterisks $\left({ }^{*} p<0.01,{ }^{* *} p<0.001\right)$ indicate significant differences

294 between the methods, while letters (c: Control, p: plug mesh method, r: rubber band method) 
295 indicate between which methods they differed in. Note: The glue method was not assessed as

296 only one out of 20 replicates successfully attached to the plug.

297

\section{3.2. Optimization of best method}

299

300 3.2.1. Success rate

301 The success rate of the best method, the plug mesh method, was assessed in a more detailed time

302 scale. Coral fragments started to attach to either the egg crate $(20 \%)$ or the plug $(6.67 \pm 6.67 \%)$

303 after $24 \mathrm{~h}$ of fragmentation (Fig. 4). From the ones unattached to anything (73.34 $\pm 6.67 \%$ ), a

304 thin layer of dead tissue could be observed falling off of the cut surface. More than half of the

305 fragments were able to attach to a surface by $48 \mathrm{~h}$ and after $72 \mathrm{~h}, 100 \%$ of the fragments were 306 attached to the plug.

309 Fig. 4. Attachment success rates of the best assessed method, the plug mesh method, over a

310 period of 72 hours. Fragments are either unattached to anything (Unattached: dark gray) or

311 attached to the egg crate wall (Egg crate: light gray) or the plug (Plug: black). Values are given

312 as means $\pm \mathrm{SE}$ of $\mathrm{N}=3$ replicates.

313

314 3.2.2. Oxygen fluxes

315 Both $P_{\text {gross }}$ and $R_{\text {dark }}$ differed significantly between the control and the plug method (one-way

316 ANOVA, $\mathrm{F}_{[1,4]}=22.52,31.79, p=0.009,0.004$, respectively). There were no significant 
317 differences for $P_{\text {net }}\left(\right.$ control $=3.78 \pm 0.57 \mu \mathrm{g} \mathrm{O}_{2}$ polyp $^{-1} \mathrm{~h}^{-1}$, plug $=6.19 \pm 0.86 \mu \mathrm{g} \mathrm{O}_{2}$ polyp$^{-1} \mathrm{~h}^{-}$

318 ; one-way ANOVA, $\left.\mathrm{F}_{[1,4]}=5.48, p=0.079\right)($ Fig. $5 \mathrm{~A})$.

319

320 3.2.3. Pulsating rate

321 On the first day, the pulsating rates were significantly lower in the fragmented plugs compared to

322 the control (one-way ANOVA, $\mathrm{F}_{[1,13]}=22.84, p<0.001$ ). After 2 days, the newly fragmented

323 coral frags had no significant difference in mean pulsating rate as the control (control $=41.78 \pm$

3240.59 , plug $=37.24 \pm 0.92$; one-way ANOVA, $\left.\mathrm{F}_{[1,15]}=4.89, p=0.043\right)$ and also for day 3 and 4

325 (one-way ANOVA, $\mathrm{F}_{[1,16]}=0.3,0.43, p=0.54,0.524$, respectively) (Fig. 5B). After 5 days of

326 fragmentation, the mean pulsating rate was significantly higher for the fragmented coral frags

$327\left(\right.$ control $=43.22 \pm 0.29$, plug $=45.20 \pm 0.29 ;$ one-way ANOVA, $\left.\mathrm{F}_{[1,16]}=8.90, p=0.009\right)$ and

328 remained significantly higher on both day 6 and 7 (one-way ANOVA, $\mathrm{F}_{[1,16]}=23.90,22.96, p<$

$3290.001,<0.001$, respectively).

330

331

332 Fig. 5. (A) Oxygen fluxes and (B) pulsating rates of Xenia umbellata fragmented with the plug

333 mesh method. Oxygen fluxes were measured after seven days of fragmentation while pulsating

334 rates were measured every $24 \mathrm{~h}$ starting from $24 \mathrm{~h}$ after fragmentation as health assessments.

$335 P_{\text {gross }}=$ gross photosynthesis, $P_{\text {net }}=$ net photosynthesis, $R_{\text {dark }}=$ dark respiration. Values of bars are

336 given as means $\pm \mathrm{SE}$ of $\mathrm{N}=3$ replicates. Asterisks $(* p<0.01, * * p<0.001)$ indicate significant

337 differences between treatments. Top bracket applies to $P_{\text {gross }}$ only.

338

339

\section{Discussion}


340 While fragmentation methods for hard corals are abundant and well described, there is a lack of

341 fragmentation techniques for studying the second biggest benthic group found on coral reefs,

342 namely soft corals. Here, we present an assessment and comparison of four different methods for

343 soft coral reproduction. Our results show that of the four assessed methods, the plug mesh

344 method, which is adapted from the tunnel cube method (Curry, 2020), is the most time- and

345 labor- effective method for easy reproduction of the soft coral X. umbellata.

346 Oxygen fluxes and pulsation rates were measured as a proxy for health of the $X$.

347 umbellata coral fragments (McGillis et al., 2009; Jantzen et al., 2013; Vollstedt et al., 2020).

348 However, no significant differences were found between the methods and the control corals,

349 suggesting that all fragments were at their respective optimal health. As such, "success rate" and

350 "fragmentation rate" were the main parameters used for the assessment of the "best method".

351 Out of the four assessed methods, the glue method, which works well for hard corals (see

352 e.g., Tilstra et al., 2017), demonstrated the lowest success rate. Only 1 out of 20 fragmented

353 colonies attached to the coral plug, albeit on the side of the plug. This failure to adhere to the

354 glue could be explained by the formation of a dead tissue layer observed after fragmentation or

355 coral derived mucus observed during fragmentation between the glue and the healthy coral

356 tissue.

357 Similar to the glue method, the rubber band method damaged the coral fragments after

358 fixation on the plug despite lowering the pressure of the rubber bands on the coral tissue to a

359 minimum. Still, various fragments were sliced in half due to the pressure put on them by the

360 rubber bands. The fragments marked as 'fail' due to slicing or no attachment to the plug surface

361 managed to attach to either the rubber band or onto different surfaces similar as the failure

362 fragments of the glue method, also resulting in no mortality. However, the success rate of the 
363 rubber band method still exceeded the glue method and was similar to the success rate of the

364 tunnel mesh method (Fig.2A). Although the rubber band method appeared to be less time

365 efficient (though not significant, Fig.2B), this method is preferred over the tunnel mesh method

366 as the mesh cannot be separated from the coral fragment when fixed to a plug. This is important

367 because algae tend to grow with ease on materials such as cotton fabrics, which can interfere

368 with experiment results (Christenson \& Sims, 2012; Gross et al., 2013). Furthermore, while the

369 coral fragments will indeed first attach to the mesh fabric, eventually they will attach to a more

370 stable surface, such as the tunnel cubes (Curry, 2020) or the egg crate used in the present study.

371 This may also explain the high success rate for the plug mesh method (Fig. 2A). Despite initial

372 attachment to the egg crate for some of the coral fragments, scraping them off the egg crate wall

373 forced the fragments to finally settle onto the plugs. This resulted in the highest success rate

374 among the four assessed methods (Fig. 2A). Additionally, when considering both success rate

375 and fragmentation time, the plug mesh method was the most labor- and time-efficient method for

376 fragging the soft coral $X$. umbellata, even after considering the additional time invested for each

377 method before and after fragmentation, e.g., mesh fabric preparation, reattachment processes,

378 and scraping off egg crate walls. Finally, considering faster recovery in pulsating rates, though

379 non-significant (Fig. 3B), and the reusability of all materials we conclude that the plug mesh

380 method is the preferred fragmentation technique for $X$. umbellata.

381 In the optimization process of the plug mesh method, more detailed data was acquired to

382 assess the attachment and recovery speed of fragments. Attachment success rates suggest that

383 fragments can attach to a surface within $24 \mathrm{~h}$ after fragmentation. However, all fragments were

384 firmly attached to the plug $72 \mathrm{~h}$ post fragmentation and was thus considered the optimum time

385 needed (Fig. 4). Attachment increased considerably after a thin layer of dead tissue was observed 
386 and removed $24 \mathrm{~h}$ post fragmentation. This dead tissue layer is likely due to epithelialization, a

387 process where epithelial cells move upwards and repair the wounded area (Meszaros \& Bigger,

388 1999). Moreover, a study that assessed the attachment abilities of the soft coral Dendronephthya

389 hemprichi reported that root-like processes (RLPs), which play a major role in the attachment

390 process, developed after four days of fragmentation (Barneah, Malik \& Benayahu, 2002). We

391 speculate that the xeniids, on the other hand, were able to attach much faster than the RLPs

392 developing $D$. hemprichi as they utilize a natural, yet unknown, adhesive material during or after

393 epithelialization has taken place (Ellis \& Sharron, 1999; Callow \& Callow, 2002; Bromley \&

394 Heinberg, 2006). Further investigation in comparing different healing rates and attachment

395 methods of an array of soft corals could shed more light on this.

396 Pulsating rates showed that a minimum of $48 \mathrm{~h}$ was needed for fragments to recover,

397 while after 6 days of fragmentation, pulsating rates were significantly higher than the control

398 (Fig. 5B). A similar pattern was observed during the main method assessment (Fig. 3B).

399 Correspondingly, the $P_{\text {gross }}$ and $R_{\text {dark }}$ were also significantly higher for the fragments than the

400 control (Fig. 5A). Whether this is the result of size difference, subsequent self-shading, or a

401 specific defense mechanism of the fragmented corals remains to be determined. Some studies

402 have demonstrated positive effects of pulsation of the $X$. umbellata as this increased gas

403 exchange between the coral and the surrounding water, thereby increasing energy and nutrient

404 acquisition (Kremien et al., 2013; Wild \& Naumann, 2013). As such, increased pulsation rates

405 could facilitate recovery of the wounded fragment by assimilating more resources. Indeed, higher

406 pulsation rates could increase $P_{\text {net }}$ and thus fixation of $\mathrm{C}$ as observed in the xeniid coral

407 Heteroxenia fuscescens (Kremien et al., 2013). However, no significant differences were found

408 in $P_{\text {net }}$ in the current study. Nonetheless, higher $R_{\text {dark }}$ and $P_{\text {gross }}$ associated with fragmented corals 
409 compared to controls suggests an enhanced metabolism, which supports the hypothesis that

410 fragments were producing and utilizing more resources, possibly for wound healing. Whether

411 this enhanced metabolism is solely due to increased autotrophy or, in combination with,

412 increased heterotrophy remains to be determined.

413

\section{5. Conclusion}

415 In conclusion, our results show that the plug mesh method is an easy, highly time- and labor-

416 efficient fragmentation method for the $X$. umbellata soft coral that could potentially be used as a

417 base for conducting essential research on diverse soft corals. It has significance in that the plug

418 mesh method makes propagating soft corals rather effortless, thus, leading to higher probabilities

419 of ex situ reproductions rather than harvesting from the wild. This could potentially result in

420 reduced disturbances of coral reef communities. The plug mesh method will also benefit

421 laboratories using soft corals as study specimens by greatly reducing the preparation time needed

422 to produce coral fragments, possibly resulting in more studies based on soft corals. As the second

423 most abundant benthic group of organisms on coral reefs, research on soft corals will increase

424 our understanding of their role in these complex ecosystems which may lead to a better

425 understanding and/or implementation of reef rehabilitation, conservation, and managing efforts. 


\section{Supplemental information}

427

428

429 Fig. S1. Image of fragments attached via the rubber band method. Rubber bands are marked with red highlights for better observation since the rubber bands that were originally used were

431 transparent.

433

434

Fig. S2. Image of fragments attached via the glue method. The glue areas are colored in red for 435 better observation.

436

\section{Acknowledgements}

the experiment. A step-by-step protocol of the plug mesh method has been uploaded to protocols.io (Kim et al., 2021).

\section{References}

443 Barneah O, Malik Z, Benayahu Y. 2002. Attachment to the substrate by soft coral fragments:

444 Desmocyte development, structure, and function. Invertebrate Biology 121:81-90. DOI: 10.1111/j.1744-7410.2002.tb00048.x.

Barton JA, Willis BL, Hutson KS. 2017. Coral propagation: a review of techniques for ornamental trade and reef restoration. Reviews in Aquaculture 9:238-256. DOI: 10.1111/raq.12135. 
449 Baum G, Januar I, Ferse SCA, Wild C, Kunzmann A. 2016. Abundance and physiology of 450 dominant soft corals linked to water quality in Jakarta Bay, Indonesia. PeerJ 4:e2625. DOI: 10.7717/peerj.2625.

452

454

456

457

458

459

460

461

462

463

464

465

466

467

468

469

470

Benayahu Y, Loya Y. 1985. Settlement and recruitment of a soft coral: why is Xenia macrospiculata a successful colonizer? Bulletin of Marine Science 36:177-188.

Borneman EH, Lowrie J. 2001. Advances in captive husbandry and propagation: An easily utilized reef replenishment means from the private sector? Bulletin of Marine Science 69:897-913.

Bromley RG, Heinberg C. 2006. Attachment strategies of organisms on hard substrates: A palaeontological view. Palaeogeography, Palaeoclimatology, Palaeoecology 232:429-453. DOI: 10.1016/j.palaeo.2005.07.007.

Callow ME, Callow JA. 2002. Marińe biofouling: A sticky problem. Biologist 49:1-5.

Chaitanawisuti N, Kritsanapuntu S. 2019. Asexual propagation of the soft corals Sinularia sp., Cladiella sp. and Sarcophyton sp. (Octocorallia: Alcyonacea) using different methods of attachment in a recirculating seawater system. Aquaculture Research 50:2047-2053. DOI: 10.1111/are.14067.

Christenson LB, Sims RC. 2012. Rotating algal biofilm reactor and spool harvester for wastewater treatment with biofuels by-products. Biotechnology and Bioengineering 109:1674-1684. DOI: 10.1002/bit.24451.

Cruz ICS, Meira VH, de Kikuchi RKP, Creed JC. 2016. The role of competition in the phase shift to dominance of the zoanthid Palythoa cf. variabilis on coral reefs. Marine Environmental Research 115:28-35. DOI: 10.1016/J.MARENVRES.2016.01.008. 
471 Curry NT. 2020. Three methods for high - volume asexual propagation of Octocorallia

472 (Alcyonaria) and Corallimorpharian soft corals. U.S. Patent Application No. 16/174,280.

473 De'Ath G, Fabricius KE, Sweatman H, Puotinen M. 2012. The 27-year decline of coral cover on 474 the Great Barrier Reef and its causes. Proceedings of the National Academy of Sciences of 475 the United States of America 109:17995-17999. DOI: 10.1073/pnas.1208909109.

476 Done TJ. 1992. Phase shifts in coral reef communities and their ecological significance. $477 \quad$ Hydrobiologia 247:121-132. DOI: 10.1007/BF00008211.

478 Ellis S, Sharron L. 1999. The Culture of Soft Corals (Order: Alcyonacea) for the Marine 479 Aquarium Trade.

480 Forsman ZH, Page CA, Toonen RJ, Vaughan D. 2015. Growing coral larger and faster: micro481 colony-fusion as a strategy for accelerating coral cover. PeerJ 3:e1313. DOI:

482

483 Gross M, Henry W, Michael C, Wen Z. 2013. Development of a rotating algal biofilm growth 484 system for attached microalgae growth with in situ biomass harvest. Bioresource 485 Technology 150:195-201. DOI: 10.1016/J.BIORTECH.2013.10.016.

486 Hoegh-Guldberg O, Pendleton L, Kaup A. 2019. People and the changing nature of coral reefs. 487 Regional Studies in Marine Science 30:100699. DOI: 10.1016/J.RSMA.2019.100699. 488 Jantzen C, Schmidt GM, Wild C, Roder C, Khokiattiwong S, Richter C. 2013. Benthic reef 489 primary production in response to large amplitude internal waves at the Similan Islands 490 (Andaman Sea, Thailand). PLoS ONE 8:1-15. DOI: 10.1371/journal.pone.0081834.

491 Johnson ME, Lustic C, Bartels E, Baums IB, Gilliam DS, Larson EA, Lirman D, Miller, 492 Margaret, W., Nedimyer K, Schopmeyer S. 2011. Caribbean Acropora Restauration Guide, 
493

494

495

496

497

498

499

500

501

502

503

504

505

506

507

508

509

510

511

512 Moberg F, Folke C. 1999. Ecological goods and services of coral reef ecosystems. Ecological 513

514 515

Best Practices for Propagation and Population Enhancement, The Nature Conservancy, Arlington, VA. :1-64.

Kim, S., Wild, C., Tilstra, A., (2021). Effective asexual propagation (fragmentation) method of the soft coral Xenia umbellata. Available at dx.doi.org/10.17504/protocols.io.bsidnca6 (accessed 20 October 2021)

Kremien M, Shavit U, Mass T, Genin A. 2013. Benefit of pulsation in soft corals. Proceedings of the National Academy of Sciences 110:8978-8983. DOI: 10.1073/PNAS.1301826110.

Maliao RJ, Turingan RG, Lin J. 2008. Phase-shift in coral reef communities in the Florida Keys National Marine Sanctuary (FKNMS), USA. Marine Biology 154:841-853. DOI: 10.1007/s00227-008-0977-0.

McClanahan TR, Cokos BA, Sala E. 2002. Algal growth and species composition under experimental control of herbivory, phosphorus and coral abundance in Glovers Reef, Belize. Marine Pollution Bulletin 44:441-451. DOI: 10.1016/S0025-326X(02)00051-6.

McGillis WR, Langdon C, Williams AJ, Loose B. 2009. O 2 -MAVS : an Instrument for Measuring Oxygen Flux. OCEANS:1-9. IEEE.

Meszaros A, Bigger C. 1999. Qualitative and Quantitative Study of Wound Healing Processes in the Coelenterate,Plexaurella fusifera:Spatial, Temporal, and Environmental (Light Attenuation) Influences. Journal of Invertebrate Pathology 73:321-331. DOI: 10.1006/JIPA.1999.4851. Economics 29:215-233. DOI: 10.1016/S0921-8009(99)00009-9.

Naumann MS, Bednarz VN, Ferse SCA, Niggl W, Wild C. 2015. Monitoring of coastal coral reefs near Dahab (Gulf of Aqaba, Red Sea) indicates local eutrophication as potential cause 
516 for change in benthic communities. Environmental Monitoring and Assessment 187:1-14.

517 DOI: $10.1007 / \mathrm{s} 10661-014-4257-9$.

518 Norström A V., Nyström M, Lokrantz J, Folke C. 2009. Alternative states on coral reefs: Beyond 519 coral-macroalgal phase shifts. Marine Ecology Progress Series 376:293-306. DOI: $520 \quad 10.3354 /$ meps07815.

521 Reaka-Kudla ML. 1997. Global biodiversity of coral reefs: a comparison with rain forests. 522 Biodiversity II: Understanding and Protecting Our Biological Resources 2: 551.

523 Tilstra A, El-Khaled YC, Roth F, Rädecker N, Pogoreutz C, Voolstra CR, Wild C. 2019.

524 Denitrification Aligns with N2 Fixation in Red Sea Corals. Scientific Reports 9:1-9. DOI: $10.1038 / \mathrm{s} 41598-019-55408-\mathrm{Z}$.

526 Tilstra A, Wijgerde T, Dini-Andreote F, Eriksson BK, Salles JF, Pen I, Osinga R, Wild C. 2017.

527 Light induced intraspecific variability in response to thermal stress in the hard coral 528 Stylophora pistillata. PeerJ 2017:1-25. DOI: 10.7717/peerj.3802.

529 Veron, J. E. (2011). Corals: biology, skeletal deposition, and reef-building. Encyclopedia of $530 \quad$ Modern Coral Reefs: Structure, Form and Process 2011:275-281.

531 Vollstedt S, Xiang N, Simancas-Giraldo SM, Wild C. 2020. Organic eutrophication increases 532 resistance of the pulsating soft coral Xenia umbellata to warming. PeerJ 2020:1-16. DOI: $533 \quad 10.7717 /$ peerj.9182.

534 Wild C, Hoegh-Guldberg O, Naumann MS, Colombo-Pallotta MF, Ateweberhan M, Fitt WK, 535 Iglesias-Prieto R, Palmer C, Bythell JC, Ortiz JC, Loya Y, Van Woesik R. 2011. Climate 536 change impedes scleractinian corals as primary reef ecosystem engineers. Marine and 537 Freshwater Research 62:205-215. DOI: 10.1071/MF10254. 
538 Wild C, Naumann MS. 2013. Effect of active water movement on energy and nutrient

539 acquisition in coral reef-associated benthic organisms. Proceedings of the National

$540 \quad$ Academy of Sciences of the United States of America 110:8767-8. DOI:

$541 \quad 10.1073 /$ pnas. 1306839110.

542 Wood E, Dipper F. 2008. What is the future for extensive areas of reef impacted by fish blasting

543 and coral bleaching and now dominated by soft corals? A case study from Malaysia .

544 Proceedings of the 11th International Coral Reef Symposium, Ft. Lauderdale, Florida, 7-11

$545 \quad$ July 2008:7-11.

546 Woodhead AJ, Hicks CC, Norström A V., Williams GJ, Graham NAJ. 2019. Coral reef

547 ecosystem services in the Anthropocene. Functional Ecology 33:1023-1034. DOI:

$548 \quad 10.1111 / 1365-2435.13331$. 


\section{Figure 1}

Illustration of (A) tunnel mesh method and (B) plug mesh method.

All components are fixed with two to four rubber bands on the sides. An extra egg crate is an important factor so that there can be water circulating through the egg crate holes. Once the coral fragment is attached to the mesh fabric or plug beneath, the egg crate and mesh fabric on top can be removed. Mesh fabrics are differentiated by diameter size of $3.5 \mathrm{~mm}$ (Large mesh: Mesh L) and $2.0 \mathrm{~mm}$ (Small mesh: Mesh S) per opening.

A

Mesh L -

Egg crate -

Mesh S -

Egg crate -

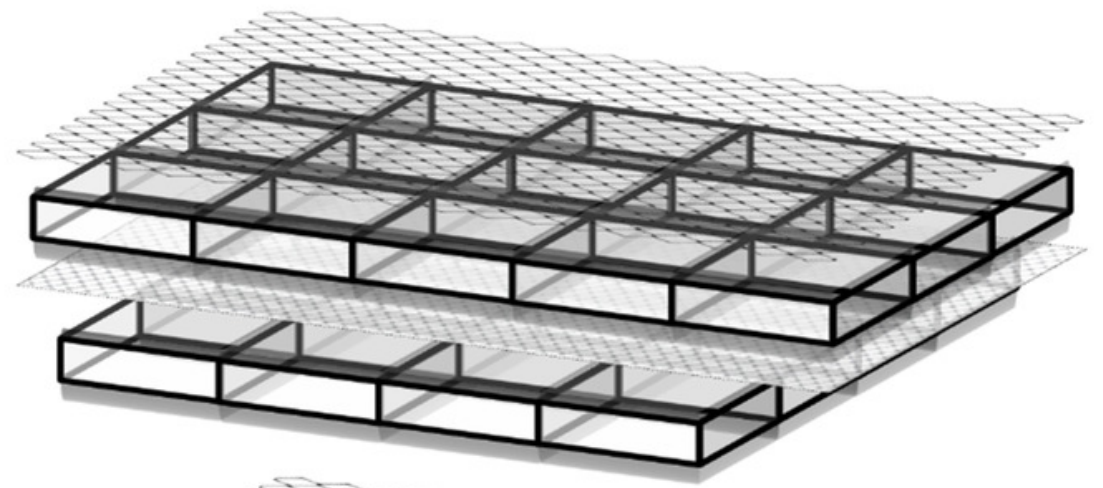

B

Mesh L -

Egg crate -

Coral Plug -

Egg crate -

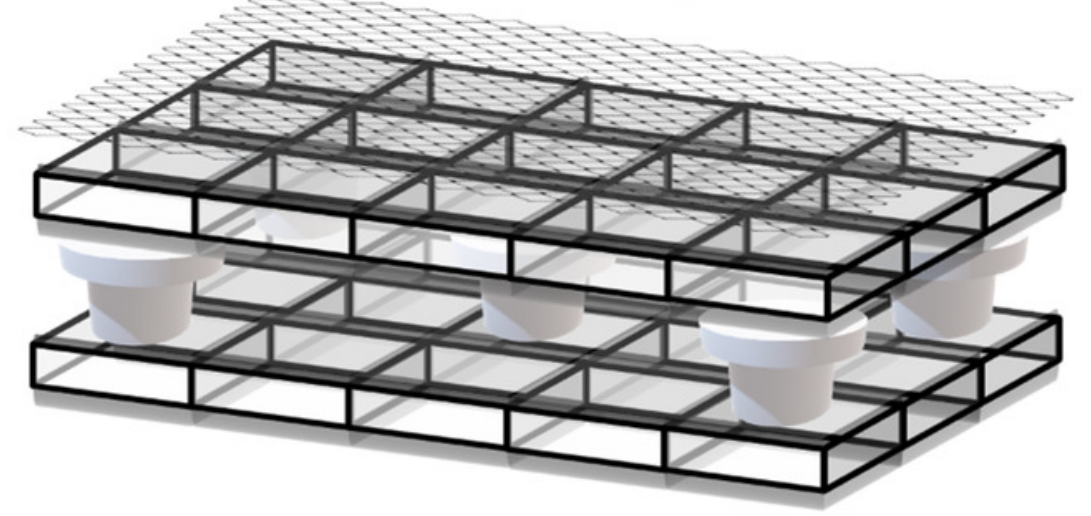


Figure 2

(A) Attachment success rate and (B) fragmentation time of four different fragmentation methods as an assessment of time- and labor-efficiency.

Four methods are the glue (Glue), rubber band (Rubber), tunnel mesh (Tunnel), and plug mesh method (Plug). Values are given as means $\pm \mathrm{SE}$ of $\mathrm{N}=3-4$ replicates. Different letters $(a, b)$ indicate significant differences between the methods (one-way ANOVA, $F_{[3,12]}=21.75, p$ $<0.001)$.

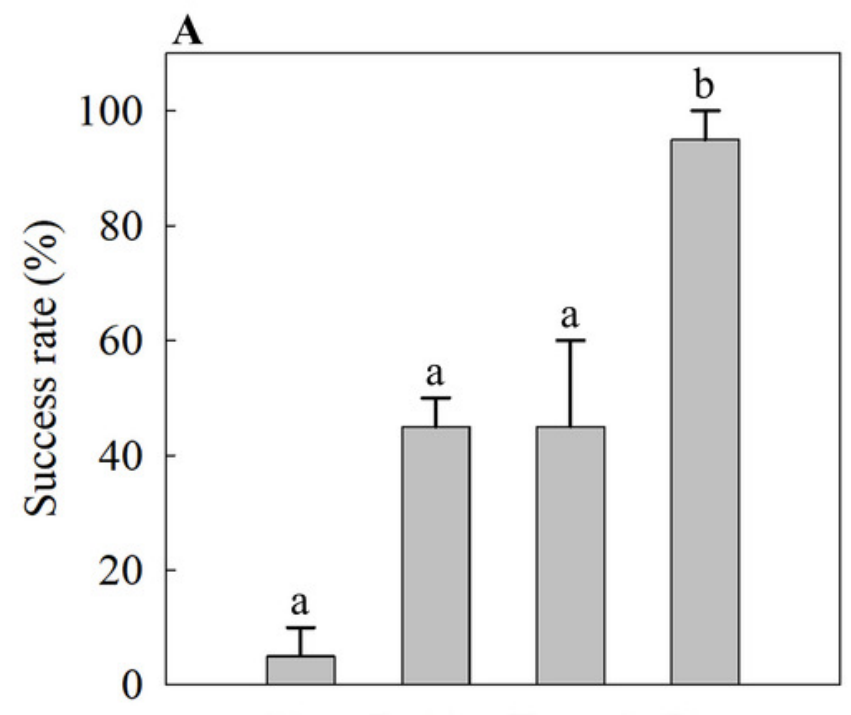

Glue RubberTunnel Plug

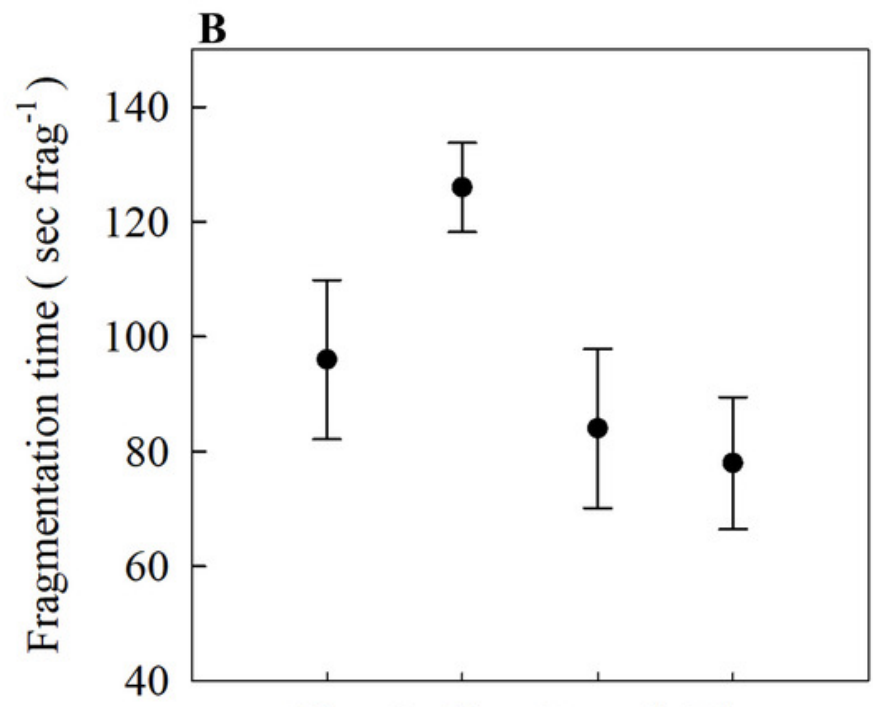

Glue RubberTunnel Plug 


\section{Figure 3}

(A) Oxygen fluxes and (B) pulsating rates measured on the twelfth-day post fragmentation as health assessments of three different fragmentation methods.

The three methods are the rubber band (rubber), tunnel mesh (tunnel), and plug mesh method (plug). $P_{\text {gross }}=$ gross photosynthesis, $P_{\text {net }}=$ net photosynthesis, $R_{\text {dark }}=$ dark respiration. Values are given as means $\pm \mathrm{SE}$ of $\mathrm{N}=3-4$ replicates. Asterisks $\left({ }^{*} p<0.01,{ }^{* *} p<\right.$ 0.001 ) indicate significant differences between the methods, while letters (c: Control, p: plug mesh method, $r$ : rubber band method) indicate between which methods they differed in. Note: The glue method was not assessed as only one out of 20 replicates successfully attached to the plug.
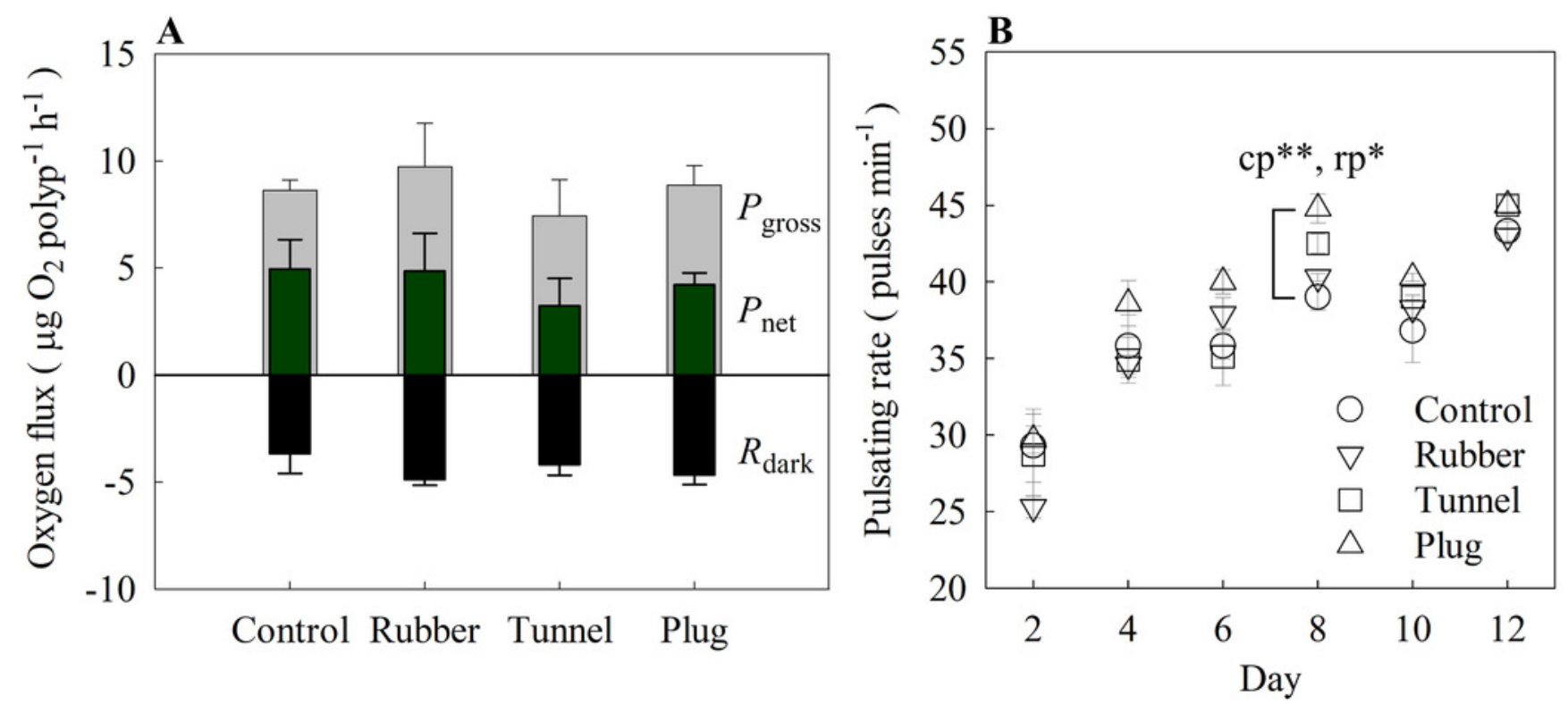
Figure 4

Attachment success rates of the best assessed method, the plug mesh method, over a period of 72 hours.

Fragments are either unattached to anything (Unattached: dark gray) or attached to the egg crate wall (Egg crate: light gray) or the plug (Plug: black). Values are given as means \pm SE of $\mathrm{N}=3$ replicates.

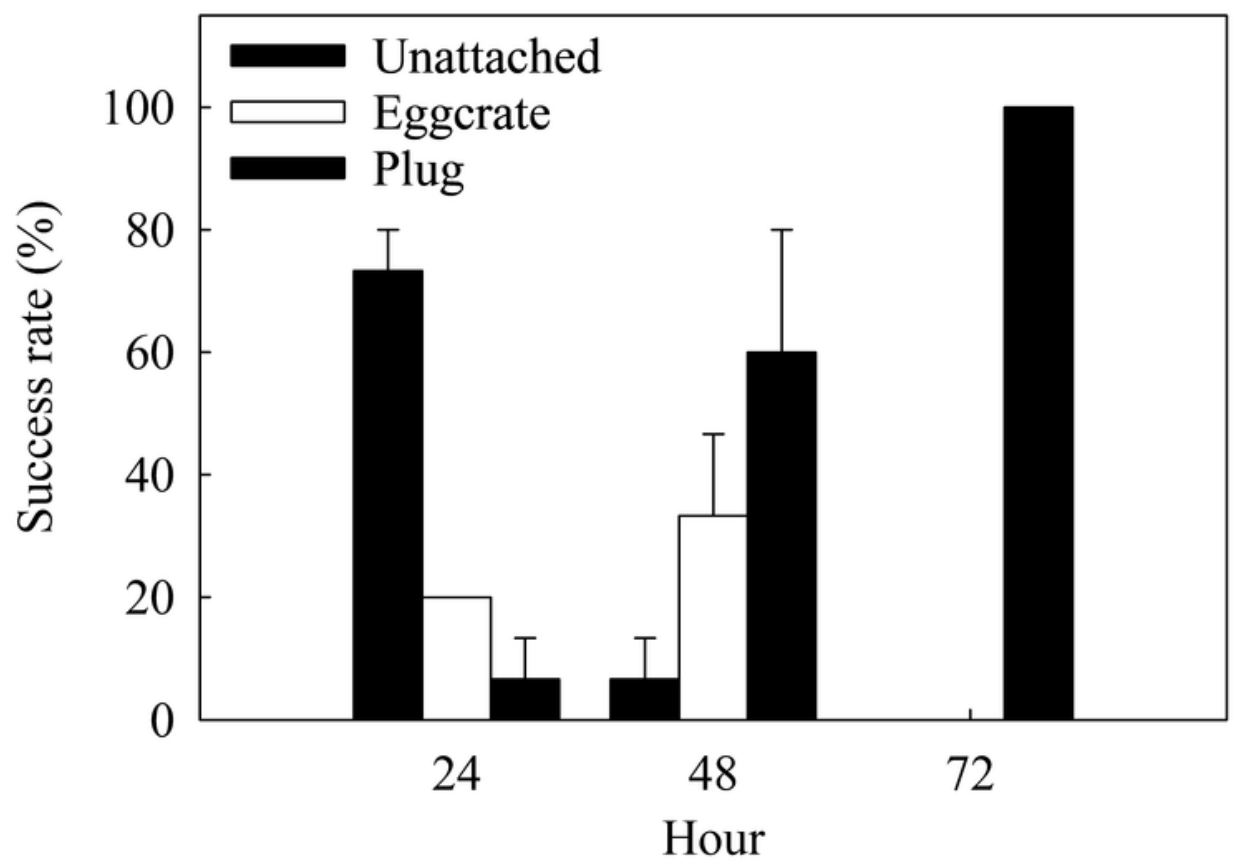


Figure 5

(A) Oxygen fluxes and (B) pulsating rates of the plug mesh method.

Oxygen fluxes were measured after seven days of fragmentation while pulsating rates were measured every $24 \mathrm{~h}$ starting from $24 \mathrm{~h}$ after fragmentation as health assessments. $P_{\text {gross }}=$ gross photosynthesis, $P_{\text {net }}=$ net photosynthesis, $R_{\text {dark }}=$ dark respiration. Values of bars are given as means $\pm \mathrm{SE}$ of $\mathrm{N}=3$ replicates. Asterisks ( ${ }^{*} p<0.01,{ }^{* *} p<0.001$ ) indicate significant differences between treatments. Top bracket applies to $P_{\text {gross }}$ only.
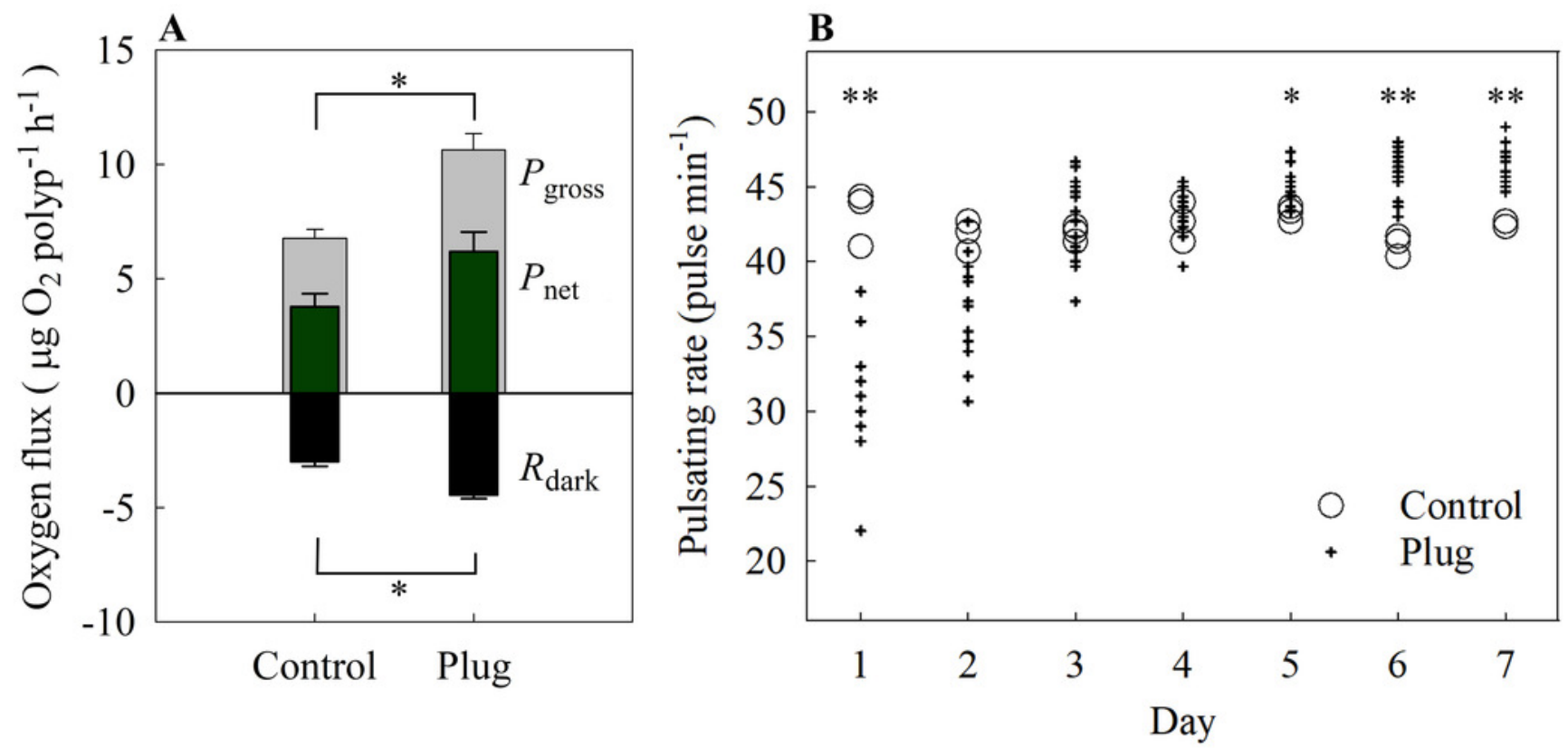